Technological University Dublin

DÜBLIN

ARROW@TU Dublin

2000-01-01

\title{
Bounds for the points of spectral concentration of sturm-liouville problems
}

Daphne Gilbert

Technological University Dublin, Daphne.Gilbert@tudublin.ie

B.J. Harris

Northern Illinois University

Follow this and additional works at: https://arrow.tudublin.ie/scschmatart

Part of the Mathematics Commons

\section{Recommended Citation}

Gilbert, D., \& Harris, B. (2000). Bounds for the points of spectral concentration of sturm-liouville problems. Mathematika, vol. 47, vol. 1-2, pg. 327-337. doi:10.1112/S002557930001593X

This Article is brought to you for free and open access by the School of Mathematics at ARROW@TU Dublin. It has been accepted for inclusion in Articles by an authorized administrator of ARROW@TU Dublin. For more information, please contact arrow.admin@tudublin.ie, aisling.coyne@tudublin.ie,gerard.connolly@tudublin.ie.

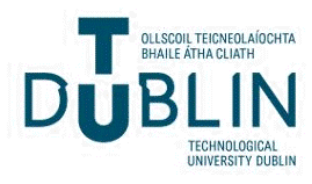




\title{
BOUNDS FOR THE POINTS OF SPECTRAL CONCENTRATION OF STURM-LIOUVILLE PROBLEMS
}

\author{
D. J. GILBERT AND B. J. HARRIS
}

$\S 1$. Introduction. We consider the spectral function $\rho_{\alpha}(\lambda)$ associated with the Sturm-Liouville equation

$$
y^{\prime \prime}+(\lambda-q(x)) y=0 \quad \text { for } x \in[0, \infty),
$$

with the boundary condition

$$
y(0) \cos \alpha+y^{\prime}(0) \sin \alpha=0 \quad \text { for some } \alpha \in[0, \pi) .
$$

We suppose that $q$ is a real-valued member of $L^{1}[0, \infty)$. We further suppose that there exist $\lambda_{0} \geqslant 0$ and functions $a(x)$ and $\eta(\lambda)$ such that

$$
\left|\int_{x}^{\infty} e^{2 i \lambda 1 / 2 t} q(t) d t\right| \leqslant a(x) \eta(\lambda) \text { for } x \geqslant 0 \text { and } \lambda \geqslant \lambda_{0} \text {, }
$$

where

(I) $a(x)$ is decreasing,

(II) $a(\cdot) \in L^{1}[0, \infty)$,

(III) $\eta(\lambda) \rightarrow 0$ as $\lambda \rightarrow \infty$.

It was shown in [9] that these conditions are satisfied, for example, if $(1+t) q(t) \in L^{1}[0, \infty)$ or if $|q| \in L^{1}[0, \infty)$ is decreasing. An example of an $L^{1}[0, \infty)$ function which is non-monotonic but for which (1.3) and (I)-(III) hold is given in $\$ 7$.

It is known that the condition $q \in L^{1}[0, \infty)$ implies that the spectral function $\rho_{\alpha}(\lambda)$ is absolutely continuous on [0, ); see [5] and [10]. In [9], an explicit formula in the form of a series was derived for $\rho_{\alpha}^{\prime}(\lambda)$ for all $\lambda \geqslant \lambda_{0}$ where $\lambda_{0}$ is computable. This formula is applicable for all $q \in L^{1}[0, \infty)$ satisfying (1.3) and (I)-(III).

In a recent paper [5], the question was considered of points of spectral concentration for operators defined by $(1.1),(1.2)$, with $q(x)$ rapidly decaying and satisfying smoothness conditions. A point of spectral concentration is defined to be a local maximum of $\rho_{\alpha}^{\prime}(\lambda)$, and is thus a value of $\lambda$ for which the spectral function is increasing relatively rapidly. We refer to [1] and [5] for a discussion of the physical significance of such points. The results of [5] lead to intervals of the form $\left[\Lambda_{0}, \infty\right)$ which are devoid of points of spectral concentration. The object is to impose conditions on $q$ which enable $\Lambda_{0}$ to be computed. The existence of $\Lambda_{0}$ is proved in [5] for $\alpha=0$ and $x q(x) \in L^{1}[0, \infty)$. Under additional conditions this existence result, together with some numerical estimates, may be used to estimate $\Lambda_{0}$. 
Our strategy is similar to that of [5] in that we provide estimates of $\Lambda_{0}$, such that $\rho_{0}^{\prime \prime}(\lambda)$ exists in $\left[\Lambda_{0}, \infty\right)$ and is of one sign. We do this by deriving the major term of $\rho_{0}^{\prime \prime}(\lambda)$ an an estimate of the remainder which holds for all $\lambda \geqslant \Lambda_{0}$. We achieve our results using a series expansion of a particular solution of the Riccati equation associated with (1.1). This solution turns out to be closely related to the spectral derivatives of interest, and enables us to extend significantly the class of potentials for which estimates of $\Lambda_{0}$ are available. We also obain analogous results for $\alpha \neq 0$; the calculation of $\rho_{\alpha}^{\prime \prime}(\lambda)$ in this case is more complicated, and we give the main term and an asymptotic estimate of the remainder which shows that ultimately there are no points of spectral concentration.

Although our results extend the class of operators for which estimates of $\Lambda_{0}$ are available, there is an important sense in which they are complementary to, and not merely an extension of, the results of [5]. This is because more stringent conditions on $q$ can be expected to lead to improved estimates for $\Lambda_{0}$, as exemplified in [5]. Thus, for $\alpha=0$ and $q$ satisfying the conditions of [5], tighter estimates of $\Lambda_{0}$ are more likely to be obtained using [5, Theorem 2] than from Theorem 1 of the present paper. We illustrate this effect in $\$ 7$, Example 2, Other examples are concerned with cases where $q$ satisfies our conditions, but not those of [5].

The authors wish to express their gratitude to the referees for a number of helpful comments.

\section{\$2. The results.}

THEOREM 1. If $\Lambda_{0} \geqslant \lambda_{0}$ is so large that, for all $\lambda \geqslant \Lambda_{0}$,

$$
32 \eta(\lambda) \int_{0}^{\infty} a(t) d t \leqslant 1,
$$

then, for all $\lambda \geqslant \Lambda_{0}, \rho_{0}^{\prime \prime}(\lambda)$ exists and satisfies

$$
\left|\rho_{0}^{\prime \prime}(\lambda)-\frac{1}{2 \pi \sqrt{\lambda}}\right| \leqslant \frac{4}{\pi} \lambda^{-1 / 2} \eta(\lambda) \int_{0}^{\infty} a(t) d t .
$$

In particular $\rho_{0}(\lambda)$ has no points of spectral concentration for $\lambda \geqslant \Lambda_{0}$.

THEOREM 2. For $\alpha \neq 0, \rho_{\alpha}^{\prime \prime}(\lambda)$ exists for all $\lambda$ sufficiently large, and

$$
\left|\sin ^{2}(\alpha) \rho_{\alpha}^{\prime \prime}(\lambda)+\frac{1}{2 \pi \lambda^{3 / 2}}\right|=O\left(\lambda^{-3 / 2} \eta(\lambda)\right) \quad \text { as } \lambda \rightarrow \infty \text {. }
$$

Thus, there are again no spectral concentration points for $\lambda$ sufficiently large.

It is convenient to gather together a number of consequences of (I), (II) and (III). 
LEMMA 1. Under the conditions of (I)-(III),

(i) $\lim _{t \rightarrow \infty} t a(t)=0$,

(ii) $\operatorname{ta}(t)^{2} \in L^{1}[0, \infty)$, (iii) $\int_{x}^{\infty} e^{2 i \lambda 1 / 2 t} t q(t) d t$ exists and is continuous as a function of $x$ for
$0 \leqslant x<\infty$.

Proof. (i) This is Abel's (or Pringsheim's) Theorem for Integrals ( $c f$. [8], p. 350).

(ii) We note that

$$
t a(t)^{2}=(t a(t)) a(t)=o(a(t)) \in L^{\mathrm{i}}[0, \infty) \text { by (i) and (II). }
$$

(iii) Using (i) and (1.3),

$$
\lim _{x \rightarrow \infty}\left|x \int_{x}^{\infty} e^{2 i \lambda / 2 t} q(t) d t\right| \leqslant \lim _{x \rightarrow \infty} x a(x) \eta(\lambda)=0 .
$$

Hence an integration by parts gives

$$
\int_{x}^{\infty} \int_{x}^{\infty} e^{2 i \lambda \lambda^{1 / 2 t}} q(t) d t d s=-x \int_{x}^{\infty} e^{2 i \lambda / / 2 t} q(t) d t+\int_{x}^{\infty} e^{2 i \lambda / 2 / 2 s} s q(s) d s,
$$

and the result follows from $(1,3)$.

\$3. The method. Let $v(x, \lambda)$ be a complex-valued solution of the Riccati equation

$$
v^{\prime}=-\lambda+q-v^{2},
$$

such that, for $\lambda>0$,

$$
v(x, \lambda)-i \lambda^{1 / 2} \rightarrow 0 \quad \text { as } x \rightarrow \infty,
$$

and

$$
v(x, \lambda)-i \lambda^{1 / 2} \in L^{1}[0, \infty) .
$$

To see that such a solution, if it exists, is unique, we may argue as follows. Suppose not; then there exist distinct $v_{1}(x, \lambda)$ and $v_{2}(x, \lambda)$ with $v_{j}(x, \lambda)=$ $i \lambda^{1 / 2}+u_{j}(x, \lambda)$ for $j=1,2$, where $u_{j}(\cdot, \lambda) \in L^{1}[0, \infty)$ and $u_{j}(x, \lambda) \rightarrow 0$ as $x \rightarrow \infty$. Substitution in (3.1) gives

$$
\left(u_{1}-u_{2}\right)^{\prime}+\left(2 i \lambda^{1 / 2}+u_{1}+u_{2}\right)\left(u_{1}-u_{2}\right)=0,
$$

whence

$$
\frac{d}{d t}\left(\left[u_{1}(t, \lambda)-u_{2}(t, \lambda)\right] \exp \left[2 i \lambda^{1 / 2} t++\int_{0}^{t} u_{1}(s, \lambda)+u_{2}(s, \lambda) d s\right]\right)=0,
$$

and after integration the contradiction follows from (3.2). 
It was shown in [9] that, for $\lambda>\lambda_{0}$ and $\alpha \neq 0$,

$$
\rho_{\alpha}^{\prime}(\lambda)=\frac{\rho_{0}^{\prime}(\lambda)}{\sin ^{2} \alpha\left(T(0, \lambda)^{2}+(S(0, \lambda)+\cot \alpha)^{2}\right.},
$$

where $S(x, \lambda)$ and $T(x, \lambda)$ denote the real and imaginary parts of $v(x, \lambda)$. An important feature of (3.4) is that it enables the boundary behaviour of the Titchmarsh-Weyl $m$-function associated with (1.1) and (1.2) to be related to the particular solution $v(x, \lambda)$ of the Riccati equation. To see this, note firstly that, from (3.4) with $\alpha=\pi / 2$, we have

$$
\frac{\rho_{\pi / 2}^{\prime}(\lambda)}{\rho_{0}^{\prime}(\lambda)}=\frac{1}{S(0, \lambda)^{2}+T(0, \lambda)^{2}}
$$

and from [4], equation (3.3),

$$
\frac{\rho_{0}^{\prime}(\lambda)}{\rho_{\pi / 2}^{\prime}(\lambda)}=\lim _{\varepsilon \rightarrow 0^{+}}|m(\lambda+i \varepsilon, 0)|^{2},
$$

where $m(\mu, 0)$ is the $m$-function corresponding to $\alpha=0$ such that

$$
S(0, \lambda)^{2}+T(0, \lambda)^{2}=\lim _{\varepsilon \rightarrow 0^{+}}|m(\lambda+i \varepsilon, 0)|^{2} .
$$

Moreover, substituting for $S(0, \lambda)^{2}+T(0, \lambda)^{2}$ in (3.4) from (3.7), and using (3.6), yields

$$
S(0, \lambda)=\frac{1}{\sin 2 \alpha}\left\{\frac{\rho_{0}^{\prime}(\lambda)}{\rho_{\alpha}^{\prime}(\lambda)}-\frac{\rho_{0}^{\prime}(\lambda)}{\rho_{\pi / 2}^{\prime}(\lambda)} \sin ^{2} \alpha-\cos ^{2} \alpha\right\},
$$

and the right-hand side is precisely the formula identified in ([6], equation (3.5)) for $\lim _{e \rightarrow 0^{+}} \Re\{m(\lambda+i \varepsilon, 0)\}$.

We can now infer from (3.3) and (3.7) that

$$
S(0, \lambda)+i T(0, \lambda)=\lim _{\varepsilon \rightarrow 0^{+}} m(\lambda+i \varepsilon, 0)
$$

for all $\lambda>\lambda_{0}$. It then follows from ([6], Lemma 1.2(i)) that

$$
\rho_{0}^{\prime}(\lambda)=\frac{1}{\pi} T(0, \lambda)
$$

from which, if $T(0, \lambda)$ is differentiable, we also have

$$
\rho_{0}^{\prime \prime}(\lambda)=\frac{1}{\pi} \frac{\partial T(0, \lambda)}{\partial \lambda} .
$$

As in [9], the main feature of our method in the present paper is a series representation of the solution $v(x, \lambda)$ of $(3.1)-(3.3)$. The series for $v(x, \lambda)$ introduced in [9] is not well suited to this type of calculation because of the complicated expression for $\lambda$ in the exponent. We therefore construct a series for a solution of (3.1), satisfying (3.2) and (3.3), which is better attuned to our needs. We note that, owing to the uniqueness of $v(x, \lambda)$, equation (3.4) and the subsequent analysis remains valid in the context of the new series, thus enabling us to identify $\rho_{0}^{\prime}(\lambda)$ in terms of $T(0, \lambda)$ and to estimate $\rho_{0}^{\prime \prime}(\lambda)$ from (3.9), 
We seek a solution of the form

$$
v(x, \lambda)=i \lambda^{1 / 2}+\sum_{n=1}^{\infty} v_{n}(x, \lambda)
$$

where $\sum_{n-1}^{\infty}\left|v_{n}(x, \lambda)\right| \in L^{1}[0, \infty)$ and goes to zero as $x \rightarrow \infty$. We set $v(x, \lambda)=$ : $S(x, \lambda)+i T(x, \lambda)$ as before. We show, in Lemma 2 below, that the series which we derive for the right-hand side of (3.10) is uniformly, absolutely convergent for $x \geqslant 0$ and $\lambda \geqslant \Lambda_{0}$, so that a term by term differentiation and rearrangement are justified. Substitution of (3.10) into (3.1) and a rearrangement gives

$$
\sum_{n=1}^{\infty}\left(v_{n}^{\prime}+2 i \lambda^{1 / 2} v_{n}\right)=q-v_{1}^{2}-\sum_{n=2}^{\infty}\left(v_{n}^{2}+2 v_{n} \sum_{m=1}^{n-1} v_{m}\right)
$$

and

$$
\begin{aligned}
v_{1}^{\prime}+2 i \lambda^{1 / 2} v_{1}+v_{2}^{\prime}+ & 2 i \lambda^{1 / 2} v_{2}+\sum_{n=3}^{\infty}\left(v_{n}^{\prime}+2 i \lambda^{1 / 2} v_{n}\right) \\
& =q-v_{1}^{2}-\sum_{n=3}^{\infty} v_{n-1}^{2}+2 v_{n-1} \sum_{m=1}^{n-2} v_{m} .
\end{aligned}
$$

We choose $v_{n}$ so that

$$
\begin{aligned}
& v_{1}^{\prime}+2 i \lambda^{1 / 2} v_{1}=q, \\
& v_{2}^{\prime}+2 i \lambda^{1 / 2} v_{2}=-v_{1}^{2}, \\
& v_{n}^{\prime}=2 i \lambda^{1 / 2} v_{n}=-\left(v_{n-1}^{2}+2 v_{n-1} \sum_{m=1}^{n-2} v_{m}\right) \quad \text { for } n \geqslant 3 .
\end{aligned}
$$

This leads to the choice

$$
\begin{aligned}
& v_{1}(x, \lambda)=-e^{-2 i \lambda \lambda^{1 / 2} x} \int_{x}^{\infty} e^{2 \lambda \lambda^{1 / 2 t}} q(t) d t, \\
& v_{2}(x, \lambda)=e^{-2 i \lambda \cdot 1 / 2 x} \int_{x}^{\infty} e^{2 i \lambda \lambda^{1 / 2 t}} v_{1}(t, \lambda)^{2} d t, \\
& v_{n}(x, \lambda)=e^{-2 i \lambda \cdot / 2 x} \int_{x}^{\infty} e^{2 i \lambda \cdot 1 / 2 t}\left(v_{n-1}^{2}+2 v_{n-1} \sum_{m=1}^{n-2} v_{m}\right) d t .
\end{aligned}
$$

REMARK 1. An alternative derivation of the results of this section, relating $\rho_{0}^{\prime}(\lambda)$ to $S(0, \lambda)$ and $T(0, \lambda)$, runs as follows; the details are given in [7]. It is known that $(1.1)$ is in the limit point case at infinity, so for $\mathfrak{I}\{\lambda\}>0$ there exists an $L^{2}[0, \infty)$ solution $y(x, \lambda)$ of $(1.1)$, which is unique up to a constant multiple. Hence, for $\mathfrak{I}\{\lambda\}>0$, the quotient $y^{\prime}(x, \lambda) / y(x, \lambda)$ is unique and satisfies (3.1). It is shown in [7] that, under conditions which are satisfied in the present context for each $\lambda \geqslant \Lambda_{0}$, the solution $v(x, \lambda)$ of (3.1) satisfying (3.2) and (3.3) may be identified with the limit of this quotient as $\lambda$ approaches the real 
axis normally; moreover, for $\alpha=0, v(0, \lambda)=\lim _{\varepsilon \rightarrow 0^{+}} m(\lambda+i \varepsilon, 0)$ and $\lim _{\varepsilon \rightarrow 0^{+}} \mathfrak{S}\{m(\lambda+i \varepsilon, 0)\}=\pi \rho_{0}^{\prime}(\lambda)$.

\$4. Properties of the series.

LEMMA 2. If $9 \eta(\lambda) \int_{0}^{\infty} a(t) d t \leqslant 1$, then $\left|v_{n}(x, \lambda)\right| \leqslant a(x) \eta(\lambda) / 2^{n-1}$ for $n=1$, $2,3, \ldots$, so that

$$
\sum_{n=1}^{\infty}\left|v_{n}(,, \lambda)\right| \in L^{1}[0, \infty) \text { and } \sum_{n=1}^{\infty}\left|v_{n}(x, \lambda)\right| \rightarrow 0 \quad \text { as } x \rightarrow \infty \text {. }
$$

Proof. This is clear from (1.3) for $n=1$. Hence, from (3.13),

$$
\left|v_{2}(x, \lambda)\right| \leqslant \int_{x}^{\infty} \eta(\lambda)^{2} a(t)^{2} d t \leqslant \eta(\lambda)^{2} a(x)\left(\int_{0}^{\infty} a(t) d t\right) \leqslant \frac{\eta(\lambda) a(x)}{2} .
$$

Suppose that the result were true for $v_{k}$, where $1 \leqslant k \leqslant n-1$, with $n \geqslant 3$. From (3.13),

$$
\begin{aligned}
\left|v_{n}(x, \lambda)\right| & \leqslant \int_{x}^{\infty}\left|v_{n-1}\right|^{2}+2\left|v_{n-1}\right| \sum_{m=1}^{n-2}\left|v_{m}\right| d t \\
& \leqslant \int_{x}^{\infty}\left\{\frac{a(t)^{2} \eta(\lambda)^{2}}{2^{2 n-4}}+\frac{2 a(t)^{2} \eta(\lambda)^{2}}{2^{n-2}} \sum_{m=1}^{\infty} \frac{1}{2^{m-1}}\right\} d t \\
& \leqslant \frac{a(x) \eta(\lambda)}{2^{n-1}} \int_{x}^{\infty}\left(\frac{a(t) \eta(\lambda)}{\left.2^{n-3}+8 a(t) \eta(\lambda)\right) d t}\right. \\
& \leqslant \frac{a(x) \eta(\lambda)}{2^{n-1}}\left(9 \eta(\lambda) \int_{0}^{\infty} a(t) d t\right),
\end{aligned}
$$

and the result follows.

It follows from Lemma 2 and the hypotheses of the theorems that $\sum_{n=1}^{\infty} v_{n}(x, \lambda)$ is uniformly, absolutely convergent for $x \geqslant 0$ and $\lambda \geqslant \Lambda_{0}$. Also, from (3.12),

$$
\begin{aligned}
\left|v_{n}^{\prime}(x, \lambda)\right| & \leqslant 2 \lambda^{1 / 2}\left|v_{n}\right|+\left|v_{n-1}\right|^{2}+2\left|v_{n-1}\right| \sum_{m=1}^{\infty}\left|v_{m}\right| \\
& \leqslant \frac{a(x) \eta(\lambda)}{2^{n-1}}\left\{2 \lambda^{1 / 2}+\frac{a(0) \eta(\lambda)}{2^{n-3}}+8 a(0) \eta(\lambda)\right\},
\end{aligned}
$$


so that $\sum_{n=1}^{\infty} v_{n}^{\prime}(x, \lambda)$ is uniformly absolutely convergent for $x \in[0, \infty)$ and $\lambda \geqslant \Lambda_{0}$. It follows that $v(x, \lambda)=i \lambda^{1 / 2}+\sum_{n=1}^{\infty} v_{n}(x, \lambda)$ does indeed represent a solution of (3.1) for $\lambda \geqslant \lambda_{0}$.

It will be necessary to consider $\partial v(x, \lambda) / \partial \lambda$ for $\lambda \geqslant \Lambda_{0}$, and to this end we define, for $n=1,2,3, \ldots$,

$$
w_{n}(x, \lambda):=\frac{\partial}{\partial \lambda} v_{n}(x, \lambda) .
$$

Lemma 3. For $\lambda \geqslant \Lambda_{0}$ and $x \in[0, \infty)$, where $\Lambda_{0} \geqslant \lambda_{0}$ is so large that $32 \eta(\lambda) \int_{0}^{\infty} a(t) d t \leqslant 1$ for all $\lambda \geqslant \Lambda_{0}$,

$$
\begin{aligned}
& w_{1}(x, \lambda)=i \lambda^{-1 / 2} e^{-2 i \lambda / / 2 x} \int_{x}^{\infty} e^{2 i \lambda \mid / 2 t} v_{1}(t, \lambda) d t, \\
& w_{2}(x, \lambda)=e^{-2 i \lambda \lambda \int_{x}^{\infty}} \int_{x}^{2 i \lambda / 2 t}\left(i \lambda^{-1 / 2} v_{2}+2 v_{1} w_{1}\right) d t, \\
& w_{n}(x, \lambda)=e^{-2 i \lambda \lambda^{1 / 2}} \int_{x}^{\infty} e^{2 i \lambda \lambda^{1 / 4 t}}\left(i \lambda^{-1 / 2} v_{n}+2 v_{n-1} w_{n-1}\right. \\
& \left.\quad+2 w_{n-1} \sum_{m=1}^{n-2} v_{m}+2 v_{n-1} \sum_{m=1}^{n-2} w_{m}\right) d t,
\end{aligned}
$$

for $n=3,4,5, \ldots$ Also,

$$
\left|w_{n}(x, \lambda)\right| \leqslant \frac{\lambda^{-1 / 2} \eta(\lambda)}{2^{n-2}} \int_{x}^{\infty} a(t) d t \quad \text { for } n=1,2,3, \ldots
$$

Proof. We use induction on $n$. We note from (3.13) that

$$
\begin{aligned}
\frac{\partial v_{1}}{\partial \lambda} & =i \lambda^{-1 / 2} x e^{-2 i \lambda \lambda^{1 / 2 x}} \int_{x}^{\infty} e^{2 i \lambda / / 2 t} q(t) d t-e^{-2 i \lambda \mid / 2 x} \int_{x}^{\infty} i \lambda^{-1 / 2} t e^{2 i \lambda / / 2 t} q(t) d t \\
& =-i \lambda^{-1 / 2} e^{-2 i \lambda \lambda^{1 / 2} x} \int_{x}^{\infty} e^{2 i \lambda / / 2 t}(t-x) q(t) d t \\
& =-i \lambda^{-1 / 2} e^{-2 i \lambda \lambda^{1 / 2}} \int_{x}^{\infty}\left(\int_{s}^{\infty} e^{2 i \lambda / 2 t} q(t) d t\right) d s
\end{aligned}
$$

by Lemma 1(iii). It follows that

$$
\left|w_{1}(x, \lambda)\right| \leqslant \lambda^{-1 / 2} \int_{x}^{\infty}\left|v_{1}(t, \lambda)\right| d t \leqslant \lambda^{-1 / 2} \eta(\lambda) \int_{x}^{\infty} a(t) d t .
$$


Our proof of the result in the case $n>1$ hinges on the equality of the mixed partial derivatives $\partial^{2} v_{n} / \partial x \partial \lambda$ and $\partial^{2} v_{n} / \partial \lambda \partial x$. To ensure this, we show that $\partial v_{n} / \partial x, \partial v_{n} / \partial \lambda$ and $\partial^{2} v_{n} / \partial \lambda \partial x$ are continuous. It is clear from (3.12) and (3.13) that $\partial v_{n} / \partial x$ is continuous for $n>1$, and it may readily be shown, by the induction hypothesis, that the same is true of $\partial v_{n} / \partial \lambda \partial x$. The continuity of $\partial v_{n} / \partial \lambda$ may also be shown by the induction hypothesis, but it requires the fact that $t v_{k}^{2}(t, \lambda) \in L^{1}[0, \infty)$ for $k=1, \ldots, n-1$. This may be shown from Lemma 2 , since

$$
\left|t v_{k}^{2}\right| \leqslant \text { const } t a(t)^{2} \in L^{1}[0, \infty)
$$

for $\lambda \geqslant \lambda_{0}$, by Lemma 1(ii).

Differentiation of (3.12) with respect to $\lambda$ gives

$$
\begin{gathered}
\frac{\partial^{2} v_{n}}{\partial \lambda \partial x}+2 i \lambda^{1 / 2} w_{n} \\
=-i \lambda^{-1 / 2} v_{n}-2 v_{n-1} w_{n-1}-2 w_{n-1} \sum_{m=1}^{n-2} v_{m}-2 v_{n-1} \sum_{m=1}^{n-2} w_{m} .
\end{gathered}
$$

The equality of $\partial^{2} v_{n} / \partial \lambda \partial x$ and $\partial^{2} v_{n} / \partial x \partial \lambda=\partial w_{n} / \partial x$ enables us to deduce from (4.1) that

$$
\begin{aligned}
& \frac{\partial}{\partial t}\left(w_{n}(t, \lambda) e^{2 i \lambda / / 2 t}\right) \\
& \quad=-\mathrm{e}^{2 i \lambda / 1 / 2}\left(i \lambda^{-1 / 2} v_{n}+2 v_{n-1} w_{n-1}+2 w_{n-1} \sum_{m=1}^{n-2} v_{m}+2 v_{n-1} \sum_{m=1}^{n-2} w_{m}\right) .
\end{aligned}
$$

An integration now gives the stated result, using Lemma 2 and the induction hypothesis. Also,

$$
\begin{aligned}
\left|w_{n}(x, \lambda)\right| \leqslant \int_{x}^{\infty} \lambda^{-1 / 2}\left|v_{n}\right|+2\left|w_{n-1}\right| \sum_{m=1}^{n-1}\left|v_{m}\right|+2\left|v_{n-1}\right| \sum_{m=1}^{n-2}\left|w_{m}\right| d t \\
\leqslant \lambda^{-1 / 2} \eta(\lambda) \int_{x}^{\infty} \frac{a(t)}{2^{n-1}}\left\{1+8 \eta(\lambda) \sum_{m=1}^{n-1} 2^{1-m} \int_{0}^{\infty} a(s) d s\right. \\
\left.\quad+4 \eta(\lambda) \sum_{m=1}^{n-2} 2^{2-m} \int_{0}^{\infty} a(s) d s\right\} d t \\
\leqslant \frac{\lambda^{-1 / 2} \eta(\lambda)}{2^{n-2}} \int_{x}^{\infty} a(t)\left\{\frac{1}{2}+16 \eta(\lambda) \int_{0}^{\infty} a(s) d s\right\} d t \\
\leqslant \frac{\lambda^{-1 / 2} \eta(\lambda)}{2^{n-2}} \int_{x}^{\infty} a(t) d t
\end{aligned}
$$

as asserted. 
\$5. Proof of Theorem 1. We recall from (3.4) and (3.10) that

$$
S(0, \lambda)+i T(0, \lambda)=i \lambda^{1 / 2}+\sum_{n=1}^{\infty} v_{n}(0, \lambda) .
$$

and, by Lemma $3, \partial T(0, \lambda) / \partial \lambda$ exists and

$$
\frac{\partial T(0, \lambda)}{\partial \lambda}=\frac{1}{2} \lambda^{-1 / 2}+\Im\left\{\sum_{n=1}^{\infty} w_{n}(0, \lambda)\right\} .
$$

So, by (3.9) and Lemma 3 , for $\lambda \geqslant \Lambda_{0}$,

$$
\begin{aligned}
\left|\rho_{0}^{\prime \prime}(\lambda)-\frac{1}{2 \pi} \lambda^{-1 / 2}\right| & =\frac{1}{\pi}\left|\Im\left\{\sum_{n=1}^{\infty} w_{n}(0, \lambda)\right\}\right| \\
& \leqslant \frac{1}{\pi} \sum_{n=1}^{\infty}\left|w_{n}(0, \lambda)\right| \\
& \leqslant \frac{\lambda^{-1 / 2}}{\pi} \eta(\lambda) \int_{0}^{\infty} a(t) d t \sum_{n=1}^{\infty} \frac{1}{2^{n-2}} \\
& =\frac{4}{\pi} \lambda^{-1 / 2} \eta(\lambda) \int_{0}^{\infty} a(t) d t
\end{aligned}
$$

as asserted.

\$6. Proof of Theorem 2. We note from (5.1) and Lemma 2 that

$$
\left\{T(0, \lambda)^{2}+(S(0, \lambda)+\cot \alpha)^{2}\right\}^{-1}=\lambda^{-1}\left(1+O\left(\lambda^{-1}\right)\right) .
$$

It follows from (5.1), (5.2) and Lemmas 2 and 3 that, for $\lambda$ sufficiently large, $\partial S(0, \lambda) / \partial \lambda$ and $\partial T(0, \lambda) / \partial \lambda$ exist, which implies that $\rho_{\alpha}^{\prime \prime}(\lambda)$ exists since

$$
\begin{aligned}
\sin ^{2} \alpha & \left\{T(0, \lambda)^{2}+(S(0, \lambda)+\cot \alpha)^{2}\right\} \rho_{\alpha}^{\prime \prime}(\lambda) \\
& =\frac{1}{\pi} \frac{\partial T(0, \lambda)}{\partial \lambda}-\frac{\frac{2}{\pi}\left\{T(0, \lambda) \frac{\partial T(0, \lambda)}{\partial \lambda}+(S(0, \lambda)+\cot \alpha) \frac{\partial S(0, \lambda)}{\partial \lambda}\right\} T(0, \lambda)}{\left\{T(0, \lambda)^{2}+(S(0, \lambda)+\cot \alpha)^{2}\right\}} \\
& =-\frac{1}{2 \pi} \lambda^{-1 / 2}+O\left(\lambda^{-1 / 2} \eta(\lambda)\right)
\end{aligned}
$$

as $\lambda \rightarrow \infty$, by (3.4) and (3.9). From (6.1) and (6.2)

$$
\sin ^{2} \alpha \rho_{\alpha}^{\prime \prime}(\lambda)=-\frac{1}{2 \pi} \lambda^{-3 / 2}+O\left(\lambda^{-3 / 2} \eta(\lambda)\right)
$$

as $\lambda \rightarrow \infty$, and the result follows. 
\$7. Examples. We consider throughout the case $\alpha=0$ so that Theorem 1 is applicable.

Example 1. Suppose that $q \in L^{1}[0, \infty)$ is monotone and continuously differentiable, with $q(x), q^{\prime}(x) \rightarrow 0$ as $x \rightarrow \infty$. An integration by parts gives

$$
\begin{aligned}
\mid \int_{x}^{\infty} e^{2 i \lambda / 2 t} q(t) d t & =\left|\left(\frac{e^{2 i \lambda \lambda^{1 / 2}} q(t)}{2 i \lambda^{1 / 2}}\right)\right|_{x}^{\infty}-\int_{x}^{\infty} \frac{e^{2 i \lambda \lambda^{1 / 2}}}{2 i \lambda^{1 / 2}} q^{\prime}(t) d t \mid \\
& \leqslant \begin{cases}q(x) \lambda^{-1 / 2} & \text { if } q(x) \geqslant 0 \text { and } q^{\prime}(x) \leqslant 0, \\
-q(x) \lambda^{-1 / 2} & \text { if } q(x) \leqslant 0 \text { and } q^{\prime}(x)>0 .\end{cases}
\end{aligned}
$$

We set $a(x):=|q(x)|, \eta(\lambda):=\lambda^{-1 / 2}$ and $\Lambda_{0}=\left(32 \int_{0}^{\infty}|q(t)| d t\right)^{2}$.

We look next at an example of a rapidly decreasing $q$ which was considered in [5].

Example 2. Suppose that $q(x)=c e^{-\pi / 4} \cos (x)$; then

$$
\begin{aligned}
\left|\int_{x}^{\infty} e^{2 i \lambda / 2 t} q(t) d t\right| & =\frac{|c|}{2}\left|\int_{x}^{\infty} e^{t\left(2 i \lambda^{1 / 2}-1 / 4+i\right)}+e^{t\left(2 i \lambda^{1 / 2}-1 / 4-i\right)} d t\right| \\
& \leqslant \frac{4|c| e^{-t \pi / 4}}{8 \lambda^{1 / 2}-5},
\end{aligned}
$$

so we may choose $a(x)=e^{-t \pi / 4}$ and $\eta(\lambda)=4|c| /\left(8 \lambda^{1 / 2}-5\right)$ to give $\Lambda_{0}=$ $(256|c|+5)^{2} / 64$.

It is interesting to compare our estimate of $\Lambda_{0}$ in Example 2 with similar estimates obtained in [5], taking into account the restrictions on $q$ which apply in each case. Setting $c=-3$, and using each of the methods developed in [5], which assume respectively that

(i) $q(x)$ is absolutely continuous and $q(x), x q(x),\{x q(x)\}^{\prime} \in L^{\prime}[0, \infty)$,

(ii) the conditions of (i) hold, and $q(x)$ is twice differentiable, values of $41^{2}$ and $15^{2}$ for $\Lambda_{0}$ were obtained. The corresponding value for $\Lambda_{0}$ using Theorem 1 is $97^{2}$. As noted above, more stringent conditions on $q$ can be expected to tighten estimates of $\Lambda_{0}$, so given that our conditions on $q$ are significantly weaker than (i) and (ii) above, this example suggests that our estimates are comparable with those of [5]. We remark in this connection that the recent development of numerical methods to identify points of spectral concentration in [2], [3] suggests that spectral concentration does actually occur in the case of Example 2, and that the theoretical estimates of both [5] and the present paper may be far from optimal.

Example 3. Suppose that

$$
q(x)=\frac{\sin (1+x)}{(1+x)^{\gamma}} \quad \text { for } 1<\gamma ;
$$


then

$$
\int_{x}^{\infty} e^{2 i \lambda / / 2} q(t) d t=\frac{1}{2 i} \int_{x}^{\infty} \frac{1}{(1+t)^{\gamma}}\left(e^{\lambda\left(2 \lambda^{1 / 2}+1\right) t+i}-e^{i\left(2 \lambda^{1 / 2}-1\right) t-i}\right) d t,
$$

and an integration by parts shows that

$$
\left|\int_{x}^{\infty} e^{2 i \lambda^{1 / 2} t} q(t) d t\right| \leqslant\left(\frac{2}{2 \lambda^{1 / 2}-1}\right)(1+x)^{-\gamma} .
$$

We set $a(x):=(1+x)^{-\gamma}$ and $\eta(\lambda):=2 /\left(2 \lambda^{1 / 2}-1\right)$, so that

$$
\Lambda_{0}=\frac{1}{4}\left(1+\frac{64}{\gamma-1}\right)^{2} \text {. }
$$

In particular, Example 3 shows that, whilst the conditions $(1+t) q(t) \in$ $L^{1}[0, \infty)$ or $q \in L^{1}[0, \infty)$ and monotone are sufficient for the applicability of Theorem 1, they are not necessary.

We note that the potentials of Examples 1 and 3 are outside the class of potentials considered in [5].

\section{References}

1. E. Brandas, M. Rittby and N. Elander. Titchmarsh-Weyl theory and its relations to scattering theory: spectral densities and cross sections; theory and applications. J. Math. Phys., 26 (1985), 2648-2658.

2. B. M. Brown, M. S. P. Eastham and D. K. R. McCormick. Spectral concentration and rapidly decaying potentials, J. Comp. Appl, Math, 81 (1997), 333-348.

3. B. M. Brown, M. S. P. Eastham and D. K. R. McCormick. Absolute continuity and spectral concentration for slowly decaying potentials. J. Comp. Appl. Math., 94 (1998), 181-197.

4. M. S, P, Eastham. The asymptotic nature of spectral functions in Sturn-Liouville problems with continuous spectra. J. Math. Analysis Appl., 213 (1997), 573-582.

5. M. S. P. Eastham. On the location of spectral concentration for Sturm-Liouville problems with rapidly decaying potential. Mathematika, 45 (1998), 25-36.

6. D. J. Gilbert and B. J. Harris. Connection formulae for spectral functions associated with singular Sturm-Liouville equations. Proc. Royal Soc. Edinburgh, 130A (2000), 25-34.

7. D, J. Gilbert and B, J. Harris. On the recovery of differential equation from its spectral functions. J. Math. Analysis AppL. 262 (2001), 355-364.

8. G. H. Hardy, A Course of Pure Mathematics. Cambridge University Press (Cambridge, 1958).

9. B. J. Harris. The form of the spectral functions associated with Sturm-Liouville problems with continuous spectrum. Mathematika, 44 (1997), 149-161.

10. E. C. Titchmarsh. Eigenfunction Expansions, Part 1 (2nd Edition). (Clarendon Press, Oxford, 1962).

Dr. D. J. Gilbert,

School of Mathematical Sciences,

Dublin Institute of Technology,

Kevin Street,

Dublin 8,

Ireland.

Dr. B. J. Harris,

Department of Mathematical Sciences,

Northern Illinois University,

DeKalb, IL 60115-2888,

U.S.A.
34B24: ORDINARY DIFFERENTIAL EQUATIONS; Boundary value problems; Sturm-Liouville theory. 